\title{
Global Integrity combining ARAIM and SBAS - Method and Operational Benefit
}

\author{
Markus Rippl, German Aerospace Center (DLR) \\ Santiago Perea, Airbus Defence And Space GmbH and Institute of Navigation, RWTH Aachen University \\ Stefan Schlüter, German Aerospace Center (DLR) \\ Christoph Günther, German Aerospace Center (DLR)
}

\section{BIOGRAPHIES}

Markus Rippl is a research fellow with the Institute of Communications and Navigation (IKN) at the German Aerospace Center (DLR). His field of work is the integrity of GNSS-based navigation using receiver-side algorithms. He received his Diploma in Electrical Engineering and Information Technology from Technische Universität München (TUM) in 2007.

Santiago Perea obtained a Master of Science in Aerospace Engineering from University of Sevilla (Spain) in 2013. In parallel, as a double degree student, he gained his Master in Mechanical and Aerospace Engineering from Illinois Institute of Technology (IIT). In 2013 he joined the staff at the Institute of Communications and Navigation at German Aerospace Center (DLR) and since 2015 he is a PhD candidate at the Institute of Navigation at RWTH Aachen University. In June 2018 he joined the GNSS Performance Team at Airbus Defence \& Space GmbH in Munich, Germany. His research focuses on new advanced algorithms for multiconstellation Advance Receiver Autonomous Integrity Monitoring (ARAIM).

Stefan Schlüter is a postdoctoral research fellow at the DLR Institute of Communications and Navigation. His research interests include ionospheric physics and GNSS augmentation. He obtained his diploma in Physics at the University of Kiel and his Ph.D. degree in Meteorology at the University of Leipzig. From 2010 to 2017 he joined the EGNOS system engineering team of the ESA Project Office in Toulouse as a seconded expert. Beginning of 2018 he returned back to DLR now working in the field of Galileo, SBAS and GBAS.

Christoph Günther studied theoretical physics at the Swiss Federal Institute of Technology (ETH) in Zurich. He completed his $\mathrm{PhD}$ in 1984 and worked in different industrial psotions at Asea Brown Boveri, Ascom and Ericsson. Since 2003, he is the director of the Institute of Communications and Navigation at the German Aerospace Center (DLR), and since 2004 he is additionally holding the Chair of Communications and Navigation at Technische Universität München (TUM).

\begin{abstract}
Today, Satellite Based Augmentation Systems (SBASs) are in transition to support dual frequency Global Positioning System (GPS) or Galileo. With this evolution comes an architectural change, mitigating some of the time-critical requirements to provision SBAS data to the user. In particular dual frequency users will no longer need fast and continuous transmission of ionospheric corrections for the safety-of-life service. Without the need to model the ionospheric delay, future SBAS may be designed with a much sparser ground network and without a GEO link.
\end{abstract}

Advanced Receiver Autonomous Integrity Monitoring (ARAIM) is a self-reliant integrity scheme that benefits from the very same update of GNSSs to dual frequency signals, and multiple constellations. The integrity information provided to ARAIM users is the Integrity Support Message (ISM) characterizing the GNSS performance with respect to integrity. ARAIM does not apply differential corrections like SBAS. The ISM message is provided to the user on a regular basis; however in contrast to GBAS or SBAS, it is significantly longer-lived and may even be provided in the form of a pre-defined dataset to be replaced only in a multiple-month time scale or on demand.

This paper extends the ARAIM integrity concept so that both GNSS measurements with an ARAIM ISM and SBAS augmented measurements can be used in a joint position solution. It discusses improvement of user integrity availability when the concept is used instead of either SBAS or ARAIM alone. To illustrate the use cases for the method, we investigate on the trade space between current geostationary-satellite (GEO) based SBAS and the proposed online ARAIM architecture; we relate the architectural commonalities and differences and finally detail the proposed combination method. The operational benefit is 
demonstrated on the basis of simulated scenarios showing the Vertical Protection Level (VPL) performance of the different user algorithms in comparison.

\section{INTRODUCTION}

Both SBAS and ARAIM provide navigation integrity to GNSS users as a service. They enable applications that rely on positioning with strict requirements on position errors and a predictable error bound. While SBAS improves both accuracy and integrity by applying differential corrections, ARAIM bounds the position error computed from uncorrected range measurements. As a concept targeting primarily multiple-constellation the accuracy of the ARAIM position solution depends on a high number of available satellites in view.

Future SBAS can benefit from the availability of dual-frequency measurements that allow the user to remove the first order ionospheric delay from the pseudorange measurements, resulting in less conservatism in the error models and tighter error bounds. Passing the responsibility of characterizing ionospheric errors from the SBAS ground segment to the user also removes an important architectural constraint of today's SBAS: The short time-to-alert (TTA) requirement that is one of the main drivers for SBAS to use GEOs for service provision.

Two different approaches can be seen in current SBAS roadmaps: On the one hand, U.S. Wide-Area Augmentation System (WAAS) is in transition to a system supporting both L1 and L5 ranging signals from the GPS constellation. The disappearance of the ionospheric threat allows provision of an integrity service supporting LPV-200 throughout the service volume of WAAS already with a single constellation. On the other hand, EGNOS as one of the more recent SBAS has always targeted augmentation of the European GNSS Galileo. In EGNOS V3, it is planned to support GPS and Galileo with signals on two frequencies by 2024 with the implementation of the V3.2 release [1].

Both architectures have in common that the temporal structure of computing and providing SBAS corrections can be simplified for DF users: Ionospheric corrections are no longer needed and all other data change more slowly over time. The integrity function of the SBAS therefore primarily overbounds the satellite orbit and clock error distribution, and provides a fault detection mechanism that guarantees timely alert to the user in case of non-nominal ranging errors.
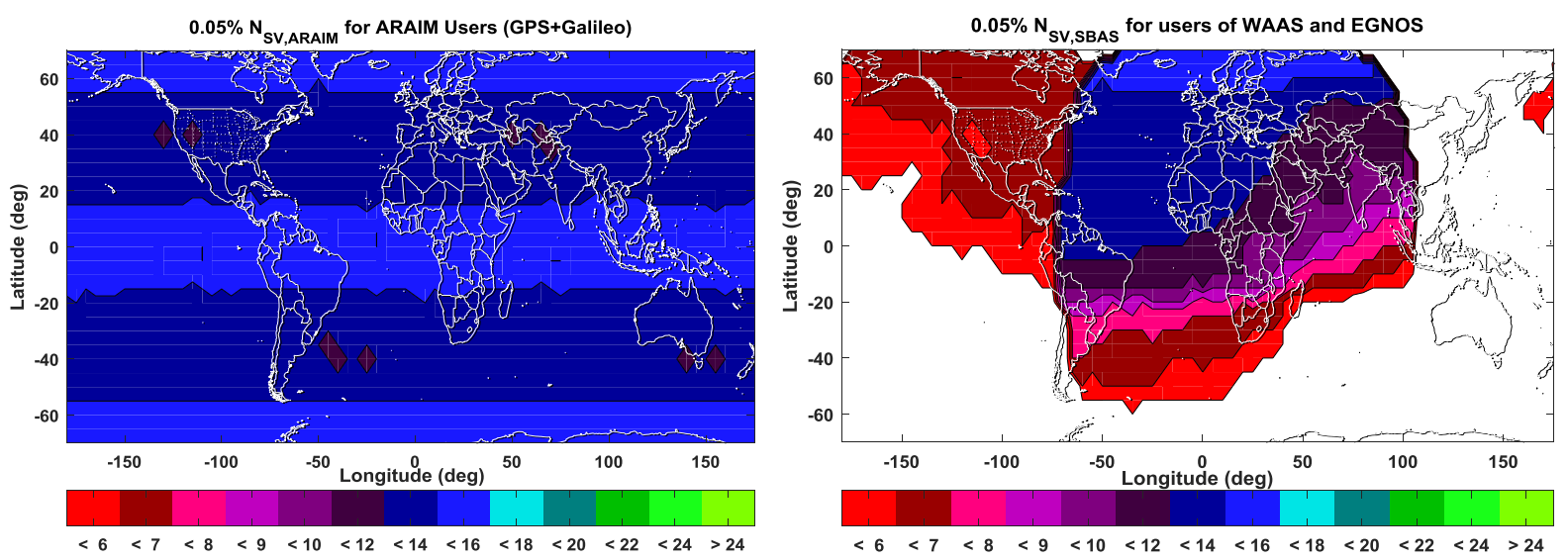

Figure 1: Usable ranging sources $\left(0.05^{\text {th }}\right.$ percentile) for users of ARAIM (left, ISM available for GPS and Galileo) and SBAS (right, only EGNOS and WAAS).

ARAIM is an integrity concept that is yet to be implemented. Its predecessor in aviation is RAIM using single frequency GPS L1 measurements. The targeted operational level for ARAIM is different: In addition to providing horizontal navigation like RAIM it will allow GNSS measurements to be used as a primary means of navigation for vertical guidance in precision approach supporting the LPV-200 requirements [2]. To accomplish this vertical aspect of its service, two prerequisites have to be fulfilled: 1) Confidence in operational performance of the core constellations, and 2) an ARAIM ground architecture that can monitor the constellation performance and provide the Integrity Support Message (ISM) to ARAIM users.

The first prerequisite is connected with the operational history of each GNSS. The objective of confirming a low enough prior fault probability can only be empirically reached by sufficient amount of independent observation data. The ISM provides two sets of parameters to overbound satellite range errors: a priori fault probabilities $\left(P_{s a t}\right.$ and $\left.P_{\text {const }}\right)$ and unfaulted error bounds $\left(\sigma_{U R A}, \sigma_{U R E}\right.$ and $\left.b_{n o m}\right)$. In an offline architecture, these parameters reflect the level of operational commitment that each constellation service provider (CSP) provides. [3, 4] design a methodology to determine the ISM parameters for GPS and Galileo unfaulted error bounds. 
The second prerequisite concerns the architecture that is used by the ARAIM ISM provider. Its function is to assess the core constellation performance in terms of nominal orbit and clock errors and signal quality. Additionally it confirms fault probability estimates for single satellites (Narrow Fault) or on system level (Wide Fault). Depending on the time interval between ISM updates and the validity period of the ISM content, different architectures can be implemented: For horizontal ARAIM (H-ARAIM), an architecture ingesting network data from external observation networks such as IGS/MGEX or SBAS reference stations can be sufficient. It provides regular confirmation on pre-defined performance parameters defined by expert opinion, and involves manual steps in processing the data similar to navigation performance reports available from GNSS and SBAS providers today [5, 6, 7]. For more stringent operations such as Vertical ARAIM (V-ARAIM) it is expected that the ISM has to be updated more frequently, and the performance characterization of the constellations has to be done in an automatic manner. Dissemination of the ISM could then be organized through any data transmission means that can provide updates to users every few hours. One option is to use the GNSS data payload itself for ISM delivery. From a manufacturer's view it features an important advantage: There is no need for a new interface and the required architectural change at avionics level is little to none.

A proposal to combine GNSS In-Band provision of ISM together with an improved navigation dataset has already been discussed at the ARAIM Technical Sub-Group of WG-C [8]. The concept foresees to provide a navigation overlay that would be used instead of the navigation information provided from the GNSS's own ground segment. The overlay would be based on independent observations of the constellation and by that, improve the performance of the orbit and clock overbounds.

\section{Use Cases}

As mentioned before, today's GNSS integrity services are diverse and multiple architectures target the same range of applications. As a consequence, different use cases are considered for the combination of ARAIM with SBAS in a unified integrity concept. We present three of them and demonstrate their relevance:

\section{Single-Constellation SBAS}

First, the current WAAS evolution roadmap does not foresee to include any other GNSS than GPS for SBAS augmentation. With the proposed combination of ARAIM and SBAS, users can improve integrity performance beyond the service that WAAS can provide by adding signals of other constellations with ARAIM support. In addition, robustness of the service is improved by reducing the impact of signal interruption or system faults.

\section{Dual/Multiple-Constellation SBAS}

EGNOS, the European SBAS, will augment Galileo and GPS in a future stage of its evolution. Our proposed concept improves the user integrity performance at the perimeter of the service volume, where the user receives signals from satellites that are not entirely monitored by the SBAS ground station network. A position solution calculated from all satellites in view, applying SBAS augmentation where available, results in better accuracy and integrity than a solution discarding the SBAS corrections. In addition, the integrity monitoring provided by SBAS can be taken into account in the integrity budget allocation of the underlying user algorithm.

\section{Integrity service unification to improve architectural degrees of freedom during service transition}

The third use case concerns longer term evolutions of SBAS, where we assume that certain economic drivers exist to phase out the use of geostationary links for dissemination. Instead, the data transmission capability of the core GNSS or other satellite systems may be used. GEO links have been chosen as the SBAS dissemination means for two technical reasons: Bandwidth and low latency. The bandwidth requirement for the dual frequency service is significantly lowered because no fast-changing ionosphere parameters have to be transmitted. The technical challenge in this transition however is the rapid integrity alerting functionality that SBAS provides in case of a fault. The current architecture relies on a low-latency channel to the airborne user through continuous reception of the GEO signal. The proposed concept mitigates the timing requirement on the SBAS Ground Segment side by providing this integrity barrier in the user receiver with ARAIM.

The improvement is a facilitation of the transition: User receivers that support the combined use of SBAS and ARAIM integrity will be able to seamlessly adapt to such changes and always work at optimal performance. Development and deployment cycles of avionics hardware can thus be decoupled from evolution steps of GNSS and SBAS. In the next section, the proposed new method is detailed and justified.

\section{METHOD}

The proposed combination of ARAIM and SBAS is based on the Multiple Hypothesis Solution Separation (MHSS) algorithm first introduced in [9]. This algorithm is adapted to plug in both the GNSS ranging measurements protected by an ISM and those augmented by SBAS. In this section, we detail the baseline ARAIM and SBAS integrity equations and the combination method. We describe the steps taken to accomplish the combination: Starting with the baseline MHSS algorithm we include 
SBAS measurements, adjust the error model to augmented ranging as provided by SBAS, and discuss the impact on the hypothesis tree and integrity performance.

In the subsequent section the new methodology is applied to a simulation that demonstrates the operational benefit of the proposed combination. We limit this analysis to the Vertical Protection Level (VPL) which is a common requirement of both SBAS and ARAIM.

\section{MHSS Vertical Protection Level}

MHSS is the user algorithm used in the ARAIM integrity concept. It provides two integrity functions: Overbounding of the nominal position error, and fault detection. Differently to Weighted Least Squares RAIM [10] no decision on the estimated fault state of one or more satellites is taken; instead it concurrently takes into account all mutually exclusive combinations of faulted and un-faulted satellites. Each of these unique combinations is a fault hypothesis, and its corresponding position solution can be computed from the subset of un-faulted satellites in that hypothesis. MHSS then combines multiple position solutions and computes an error bound that encloses the true position as long as one of the considered hypotheses is correct. The selection of hypotheses to be computed within MHSS is made such that the probability of the correct hypothesis being among those not computed is below a fraction of the available integrity budget.

MHSS relies on the provision of an Integrity Support Message (ISM) that contains information on the GNSS constellation performance as defined in Table 1 . These parameters allow the receiver to compute $\sigma_{U R A, i}, \sigma_{U R E, i}, b_{n o m, i}$ and $P_{\text {sat }, i}$ for each satellite $i$; and $P_{\text {const, } j}$ for each constellation $j[2]$.

In the following equations we define the following nomenclature: Definitions relating to hypothesis $k$ have the hypothesis index in parantheses as a superscript, e.g. subset geometry $\boldsymbol{G}^{(k)}$. Quantities relating to a satellite $i$ have a subscript index, e.g. the standard deviation of the $i^{\text {th }}$ satellite pseudorange error distribution $\sigma_{i}$. Similarly we denote constellation related quantities with a subscript $j$.

Table 1: ISM Parameters [2]

\begin{tabular}{|c|c|}
\hline Identifier & Description \\
\hline$\sigma_{U R A, i}$ & Standard deviation of clock and ephemeris error overbound of satellite $i$ \\
\hline$\sigma_{U R E, i}$ & Standard deviation of clock and ephemeris error of satellite $i$ used for accuracy and continuity \\
\hline$b_{n o m, i}$ & Maximum nominal bias for satellite $i$ used for integrity \\
\hline$P_{\text {sat }, i}$ & Prior probability of a satellite fault, Satellite $i$, per approach \\
\hline$P_{\text {const }, j}$ & Prior probability of a fault affecting multiple satellites in constellation $j$ per approach \\
\hline
\end{tabular}

This dataset allows MHSS to perform three functions [11]:

- Selection of the fault hypotheses to be taken into account for computation of the subset solutions

- Computation of the error bounds for each subset solution and the solution separation

- Fault detection and exclusion (FDE) for single or multiple satellite faults

The first function is the selection of fault hypotheses to be monitored for faults by MHSS. It assigns each satellite a binary fault state: Either the satellite is within or outside its nominal specifications. If its state is "non-faulty", the nominal performance parameters $\sigma_{U R A, i}, \sigma_{U R E, i}$, and $b_{n o m, i}$ correctly describe the true range error distribution of the satellite signal received at the user. If its state is faulty, the user range error is arbitrary and any navigation solution including this satellite may result in a position error outside the admissible error bound.

The total number of fault hypotheses using a geometry size of N satellites is $2^{N}$ and MHSS needs to select which of the fault hypotheses it has to include in the computation of the integrity equations. For a given hypothesis $k$ with a set $\boldsymbol{S}^{(k)}$ indexing satellites in fault state, we can assign a prior probability derived from the individual satellite fault probabilities

$$
P_{\text {prior }}^{(k)}=\prod_{i \in \boldsymbol{S}^{(k)}} P_{\text {sat }, i} \prod_{i \notin \boldsymbol{S}^{(k)}} 1-P_{\text {sat }, i}
$$

With a $P_{\text {sat }}^{i} \ll 1$ (a typical value is $P_{\text {sat }}^{i}=1 \times 10^{5}$ ), hypotheses containing multiple satellites in $\boldsymbol{S}^{(k)}$ have a very low prior probability. The selection of hypotheses not to be considered is made such that their sum probability is only a fraction of the allocated integrity risk $P_{H M I}$. It is then acceptable to leave these subsets un-monitored. All remaining subsets are included in 
the computation of a position solution together with a protection level, so that the true position lies within the protection level at a probability of $1-P_{H M I}$.

The second function of MHSS is the computation of the error bounds (Protection Level) for each of the subset geometries. The error bound is an error propagation of nominal distributions of the smoothed pseudorange measurement errors, worst case combinations of ranging biases at the user level, and a solution separation term modeling the accuracy of the fault monitors built into ARAIM.

The nominal smoothed pseudorange error is overbounded by a Gaussian distribution:

$$
\sigma_{i}^{2}=\sigma_{U R A, i}^{2}+\sigma_{\text {tropo }, i}^{2}+\sigma_{c n m p, i}^{2}
$$

where $\sigma_{U R A, i}^{2}$ denotes the overbound of satellite orbit and clock errors projected into the worst-case user line-of sight, $\sigma_{\text {tropo, } i}^{2}$ is the worst case tropospheric residual error overbound after applying the tropospheric error model and $\sigma_{c n m p, i}^{2}$ denotes the multipath and tracking noise at user level for an airborne user [12].

The vertical projection of the combination of nominal errors from all satellites in (k), using the geometry $\boldsymbol{G}^{(k)}$ and weighting matrix $\boldsymbol{W}^{(k)}$, is then

$$
\left(\sigma_{v}^{(k)}\right)^{2}=\left|\left(\boldsymbol{G}^{(k)^{T}} \boldsymbol{W}^{(k)} \boldsymbol{G}^{(k)}\right)^{-1}\right|_{3,3}
$$

The Gaussian overbound of the nominal position error in one of the position axes allows to quantify an upper bound on the probability that the error is above a certain threshold. Its inverse tail distribution function is used to compute an inflation factor related to the assigned integrity risk,

$$
K_{v}^{(k)}=Q^{-1}\left(P_{H M I}^{(k)}\right)
$$

Biases caused by nominal signal deformation or phase center variations of transmit and receive antenna are modeled with a bias $b_{n o m, i}$. ISM provides this bias magnitude to describe the minimum bias that could be observed by the ARAIM monitoring network, and consequently the user must assume this maximum bias magnitude to be present when computing the error bound. Moreover, the orientation of the biases in each of the satellite ranging measurements is unknown and as such they are aligned such as to generate the maximum impact in each direction of the local coordinate system. With the projection matrix $S=$ $\left(G^{T} W G\right)^{-1} G^{T} W$ derived from the Geometry $G$ and the Weighting Matrix $W$, the worst-case combination of biases in the vertical axis is

$$
b_{v}=\sum_{\text {all } i}|\boldsymbol{S}|_{3, i} \cdot b_{n o m, i} .
$$

In the above equation we have left out the index denoting the subset $(k)$ for clarity.

The last term contributing to the error bound is the solution separation for each subset. It accounts for the sensitivity of the solution separation test and is defined as

$$
T_{v}^{(k)}=K_{f a, v}^{(k)} \sigma_{s s, v}^{(k)}
$$

where the distribution of the solution separation is computed from the projection of the covariance into the vertical axis,

$$
\sigma_{i, s s, 3}=\boldsymbol{e}_{3}^{T}\left(\boldsymbol{S}^{i}-\boldsymbol{S}^{0}\right) \boldsymbol{C}_{a c c}\left(\boldsymbol{S}^{i}-\boldsymbol{S}^{0}\right)^{T} \boldsymbol{e}_{3}
$$

The inflation factor $K_{f a, i}$ corresponds with the partial continuity risk allocated to each test.

The three contributions to the VPL for each hypothesis $(k)$ can be written together as

$$
V P L^{(k)}=K_{v}^{(k)} \sigma_{v}^{(k)}+\sum_{\text {all i in }(k)}\left|\boldsymbol{S}^{(k)}\right|_{3, i} \cdot b_{\text {nom }, i}+T_{v}^{(k)}
$$

Each partial VPL can be adjusted through the allocation of integrity risk for this hypothesis. The solution VPL is defined by

$$
P_{H M I} \geq 2 Q\left(\frac{V P L-b_{v}^{(0)}}{\sigma_{v}^{(0)}}\right)+\sum_{k=1 . . N} P_{\text {prior }}^{(k)} \cdot Q\left(\frac{V P L-b_{v}^{(k)}-T_{v}^{(k)}}{\sigma_{v}^{(k)}}\right)+P_{\text {unobserved }}
$$


A detailed description of the reference algorithm is described in [11] and the ARAIM Algorithm Definition Document appended to the WG-C Milestone 3 report [2]. Relating to the present work we can see a similarity with SBAS protection level computation which is presented in the subsequent paragraph.

\section{SBAS Vertical Protection Level}

In this paragraph we review the Dual Frequency SBAS VPL equations currently under finalization in EUROCAE WG-62's Minimum Operational Performance Specification for Galileo / GPS / SBAS Airborne Equipment [13]. The error of the smoothed, corrected pseudorange measurements can be overbounded with

$$
\sigma_{i, D F S B A S}^{2}=\sigma_{D F C, i}^{2}+\sigma_{U I R E, i}^{2}+\sigma_{\text {tropo }, i}^{2}+\sigma_{a i r, i}^{2}
$$

Two of the terms in this equation have already been introduced in the ARAIM equations: $\sigma_{\text {tropo,i }}^{2}$ and $\sigma_{\text {air }, i}^{2}$ overbound the residual tropospheric error and the airborne multipath and tracking noise for smoothed measurements. Those errors are uncorrelated between different users and thus cannot be corrected by SBAS.

$\sigma_{D F C, i}^{2}$ is the model variance for the residual error associated to SBAS corrections ([13], A.3) and is computed from the SBAS Dual Frequency Ranging Error Indicator (DFREI) $\sigma_{D F R E}$, the user location factor $\delta_{D F R E}$ and the degradation parameters $\varepsilon_{c o r r}$ and $\varepsilon_{e r}$. The user ionospheric residual error, i.e. higher-order ionospheric delay that cannot be corrected by the dual frequency linear combination, is modeled as

$$
\sigma_{U I R E}=\frac{40}{261+\left(E l_{[\operatorname{deg}]}\right)^{2}}+0.018[\mathrm{~m}]
$$

This is an elevation dependent function that, for satellites at elevations above 2 degrees, results in a maximum error of $\sim 17 \mathrm{~cm}$ (Figure 2).

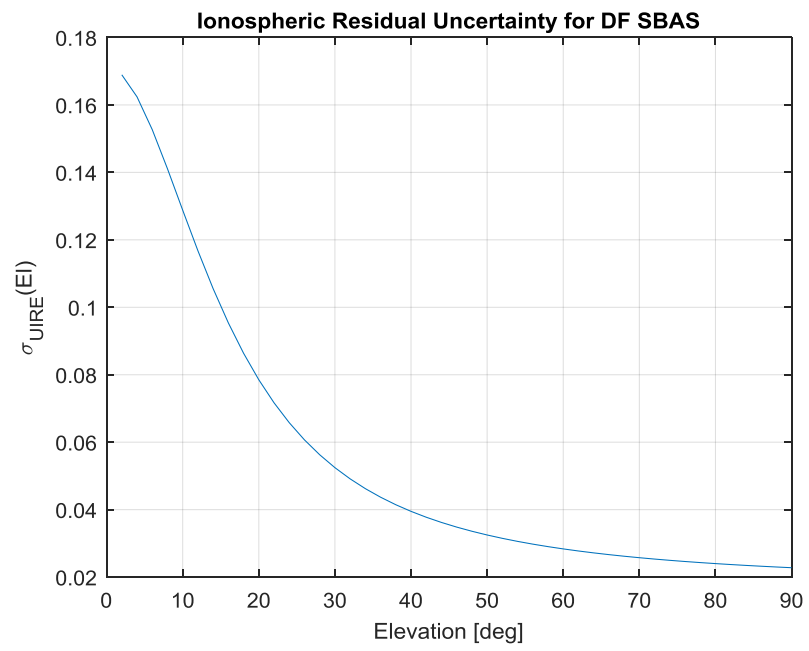

Figure 2: Ionospheric Residual Uncertainty for Dual Frequency SBAS [13]

The VPL for an SBAS augmented position solution can be computed from

$$
V P L_{D F S B A S}=K_{v, S B A S} \cdot \sigma_{v}
$$

with the covariance matrix defined from the vector of satellite ranging error standard deviations

$$
\boldsymbol{W}=\operatorname{diag}\left(1 / \boldsymbol{\sigma}_{\boldsymbol{i}, \boldsymbol{D F S} \boldsymbol{B} \boldsymbol{A} \boldsymbol{S}}\right)
$$

and, assuming uncorrelated measurement errors, the position covariance defined by the two-constellation geometry matrix and the ranging covariance 


$$
\left[\begin{array}{ccccc}
\sigma_{E}^{2} & & & & \\
& \sigma_{N}^{2} & & & \\
& & \sigma_{v}^{2} & & \\
& & & \sigma_{t 1^{2}} & \\
& & & & \sigma_{t 2}^{2}
\end{array}\right]=\left(\boldsymbol{G}^{T} \cdot \boldsymbol{W} \cdot \boldsymbol{G}\right)^{-1}
$$

The inflation factor $K_{v, S B A S}=5.33$ corresponds with a two-sided tail probability $P_{H M I}=2 \cdot Q(5.33) \approx 10^{-7}$.

\section{Consolidation of ARAIM and SBAS Protection Level Equations}

The protection level equations presented in the previous section on MHSS and SBAS have obvious commonalities when it is considered that SBAS provides an integrity barrier against faulted satellites already on system level; i.e. the range measurements in the SBAS geometry can always be considered fault-free. The proposed method uses the MHSS integrity equations together with an adaptation of the error model in case of SBAS corrected measurements. It is shown that the resulting MHSS protection level converges with the SBAS protection level under a set of assumptions. With this identity we can exchange the SBAS equations with the MHSS equations for geometries fully augmented by SBAS, but moreover it is possible to combine satellite pseudoranges with and without SBAS augmentation into a joint geometry with a valid protection level equation. We illustrate this consolidation in the following under the assumption that all visible satellites are augmented by SBAS. In that case, the integrity equation becomes ident with the SBAS equation.

The first adaptation relates to the prior fault probability of satellites with SBAS augmentation available. Assuming the prior fault probability $P_{\text {sat.SBAS }}^{i}=0 \forall i \in S B A S$, the MHSS set of hypotheses reduces to the fault-free hypothesis. The VPL equation is then

$$
V P L_{M H S S, S B A S}=K^{(0)} \sigma_{v}^{(0)}+\sum_{\text {all } i}|S|_{3, i} b_{n o m, i}
$$

Because no integrity budget has to be allocated into fault hypotheses the inflation factor used on the Gaussian overbound can be set to the full integrity budget

$$
K^{(0)}=Q^{-1}\left(0.5 \cdot P_{H M I}\right)=5.33
$$

for $P_{H M I}=10^{-7}$ (for simplicity we assume no allocation to horizontal integrity in this work).

A similar adaptation can be made for constellation faults. Satellites with SBAS augmentation are considered monitored against constellation faults and are not excluded in the constellation fault hypothesis of the constellation they belong to. If all satellites of a given constellation are processed with SBAS, the user receiver can deselect the constellation fault hypothesis and leave it unmonitored.

The second adaptation relates to the nominal bias term introduced in equation (5). Nominal GNSS signal deformation causes measurement biases at the receiver level, and the bias magnitude depends on the receiver configuration, i.e. RF bandwidth, correlator types and the correlator spacing. In ARAIM the nominal magnitude of such biases is described with the $b_{\text {nom }}$ parameter and is conservatively added among all satellites contributing to the position solution.

SBAS provides differential corrections determined from a network of precise reference stations operated in a controlled environment, and biases impacting aviation users are observed also at the reference stations. The differential corrections therefore include ranging errors introduced by such biases. Consequently, the nominal integrity bias can be neglected in the VPL equation for all satellites corrected by SBAS. For a fully augmented geometry, the VPL equation is now reduced to

$$
V P L_{M H S S, S B A S}^{*}=K^{(0)} \sigma_{v}^{(0)} \text {. }
$$

The form of equation (17) is now identical with the SBAS VPL equation (12). The error model behind $\sigma_{v}^{(0)}$ can now be adjusted to overbound the range error ofa differentially corrected pseudorange. From Equation (NN) it follows that the satellite orbit and clock errors can be overbounded in the SBAS case by $\sigma_{D F C, i}^{2}$. In addition we include a residual ionospheric error $\sigma_{U I R E, i}^{2}$ that currently has no equivalent in the MHSS error model.

The third adaptation is therefore an adjustment of the nominal error overbound for satellites corrected with SBAS. In the case of an SBAS-only geometry we have

$$
\sigma_{i, S B A S}^{2}=\sigma_{D F C, i}^{2}+\sigma_{U I R E, i}^{2}+\sigma_{\text {tropo }, i}^{2}+\sigma_{\text {air }, i}^{2} \forall i
$$

and the VPL computed with the MHSS algorithm matches the one computed with the SBAS Dual Frequency equations. 


\section{RESULTS}

This section revisits the use cases introduced above and presents simulation results that confirm the suggested improvements. In our simulation, the Stanford GPS Lab's MatLab Algorithm Availability Simulation Tool [14] was used to compute the user geometries both for ARAIM and for the comparative SBAS case. Nominal constellations consisting of 24 GPS and 24 Galileo satellites were simulated during a ten day period to ensure full geometric diversity. A user grid of 5 by 5 degrees spacing defines the service volume. The summary of the simulation parameters is reproduced in Table 2 for the first two use cases (WAAS and EGNOS scenarios).

Table 2: Simulation parameters

\begin{tabular}{|c|c|c|}
\hline Parameter & UC1 & UC2 \\
\hline GNSS Constellations & \multicolumn{2}{|l|}{ Nominal 24 GPS+24 Galileo } \\
\hline SBAS G/S & $38 \mathrm{WRS}$ & 41 RIMS \\
\hline SBAS S/S & 3 GEOs (SM-9, CRW, CRE) & 2 GEOs (5B, SES-5) \\
\hline SBAS scope & GPS only / Dual Frequency & GPS + Galileo / Dual Frequency \\
\hline SBAS UDRE model & \multicolumn{2}{|l|}{ constant } \\
\hline ARAIM URA & \multicolumn{2}{|l|}{$1.5 \mathrm{~m}$} \\
\hline SBAS URE & \multicolumn{2}{|l|}{$1.0 \mathrm{~m}$} \\
\hline$b_{\text {nom }}$ & \multicolumn{2}{|l|}{$0.75 \mathrm{~m}$} \\
\hline$P_{\text {sat }}$ & \multicolumn{2}{|l|}{$10^{-5}$} \\
\hline$P_{\text {const }}$ & \multicolumn{2}{|l|}{$10^{-8}$} \\
\hline Duration & \multicolumn{2}{|l|}{$10 \mathrm{~d}$} \\
\hline Time interval & \multicolumn{2}{|l|}{$15 \mathrm{~min}$} \\
\hline User grid & \multicolumn{2}{|l|}{$5 \times 5 \mathrm{deg}$} \\
\hline$P_{H M I}$ & \multicolumn{2}{|l|}{$9.8 \cdot 10^{-8}$ Vert. $/ 2 \cdot 10^{-9}$ Horiz. } \\
\hline PA Mode (SBAS) & \multicolumn{2}{|l|}{ enabled } \\
\hline
\end{tabular}

Here the simulated SBAS applies a heavily simplified model to determine the availability of the SBAS correction for a given user and satellite, and the computation of the UDRE. The SBAS augmentation is considered available when the satellite has been in view of the SBAS reference stations for a given time, and any of the SBAS GEOs is in view of the user. The resulting performance level is thus not representative w.r.t. the targeted performance levels of the future DF SBAS. However this simplified model is sufficient to demonstrate the comparison of the SBAS equations with the MHSS equations adapted to include $S B A S$.

\section{Single Constellation SBAS}

The first use case demonstrated by simulation is the Single Constellation (SC) SBAS. In our example we simulate a simplified DF WAAS with three GEOs. In this scenario, WAAS provides only augmentation for GPS satellites while Galileo integrity is available using an ISM and ARAIM.

In Figure 3, a comparison of the 0.995 quantiles of standard ARAIM VPL (left) and SBAS VPL (right) lets us observe that augmentation of only a single constellation already results in a significantly higher integrity performance. It increases even more if SBAS measurements are used together with ARAIM (middle) and the user can process measurements from all satellites in view. This can be seen from the area within the footprint of the GEOs where the combined performance outperforms the single constellation SBAS. 

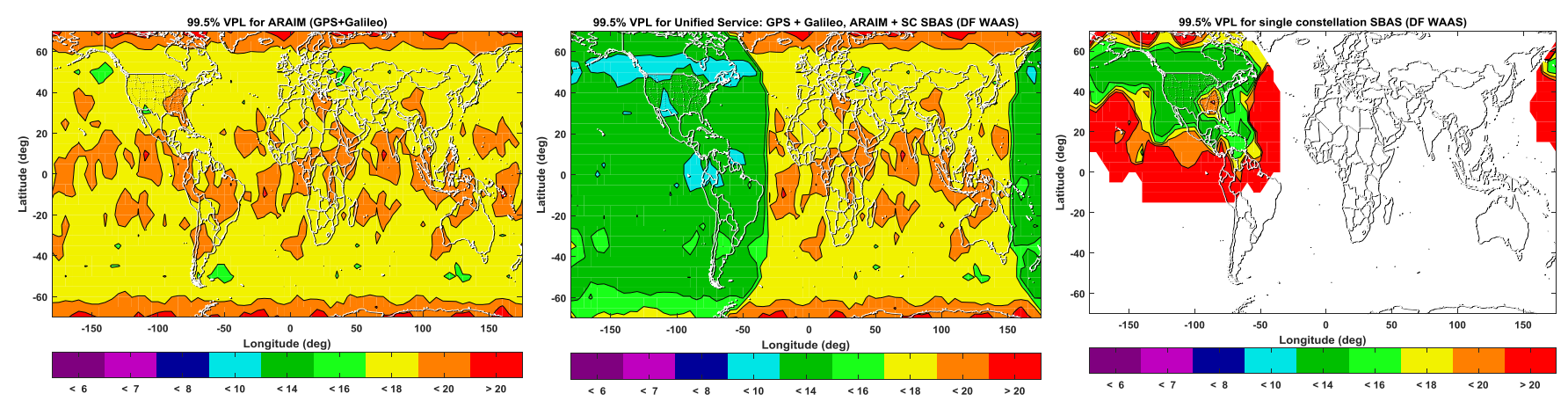

Figure 3: VPL performance with a combination of Single Constellation SBAS (e.g. WAAS) and Dual Constellation ARAIM

It is emphasized that the absolute performance levels of all three results are a function of the assumptions and should not be read as an estimate of the actual performance of the three services.

\section{Dual Constellation SBAS}

In the second use case we simulate the EGNOS SBAS as an exemplary dual-frequency, dual constellation (DFDC) augmentation system. In this scenario, users can always process both GPS and Galileo satellites with augmentation within the SBAS service volume. The performance gap between ARAIM and DFDC SBAS is therefore significantly higher. A combination of the SBAS augmentation with the MHSS user algorithm therefore does not result in a better performance in any of the simulated geometries (Figure 4). However an operational advantage is that a single integrity algorithm now provides the optimal performance at all locations, irrespective of the availability of the SBAS augmentation. This seamless service can be an operational advantage even more within the Service Volume if it helps improving continuity if the SBAS service is interrupted unexpectedly, e.g. by terrain.
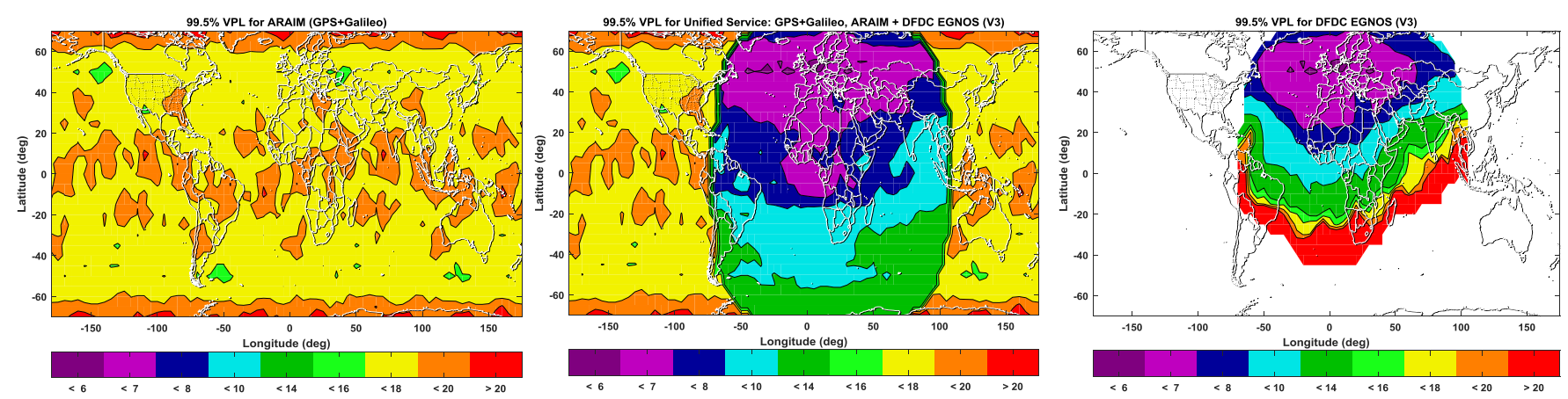

Figure 4: VPL Performance with a combination of a Dual Frequency SBAS (e.g. EGNOS V3) and ARAIM

\section{Integrity service unification for service transition}

A third use case was previously described regarding the architectural choices in transitioning to future evolutions of SBAS. Today SBAS provides fast corrections and integrity information through a GEO, but different architectures might be desirable in the future. For example, SBAS corrections and nominal integrity models could be provided through a dissemination channel with higher latency, e.g. as part of the GNSS navigation payload. Both these data are strongly correlated over time and need less update rate than a GEO can provide. If this transition is implemented however the fast integrity flagging mechanism is no more available, and fault detection would be in the responsibility of the user.

The proposed MHSS based algorithm could implement such a function with a minimal change in the error model assumptions: While the nominal error model parameters provided by SBAS are applied together with the corrections, it is no longer acceptable to assume fault-free satellites. Consequently the satellite fault probability would be non-zero and we re-introduce fault hypotheses, but using the SBAS nominal error models for the satellites. Depending on the monitoring function provided by SBAS, this $P_{\text {sat }, S B A S}$ could be different from the $P_{\text {sat }}$ provided by ISM.

We illustrate this use case by an example of a system including an SBAS and an ISM valid for both constellations. In Figure 5 the left plot shows again the unified MHSS approach when a DFDC SBAS is available and provides monitoring of the satellites. In the right plot, the FDE function of the SBAS is not available and we assume a $P_{\text {sat,SBAS }}=10^{-5}$ per approach for the satellites. Still the measurements can be differentially corrected and the nominal biases are set to zero. The interesting 
result in this analysis is that the performance decrease is minimal, but the architectural change is significant: The right scenario could be operated without a GEO if the SBAS data would be transmitted e.g. through the GNSS navigation bits.
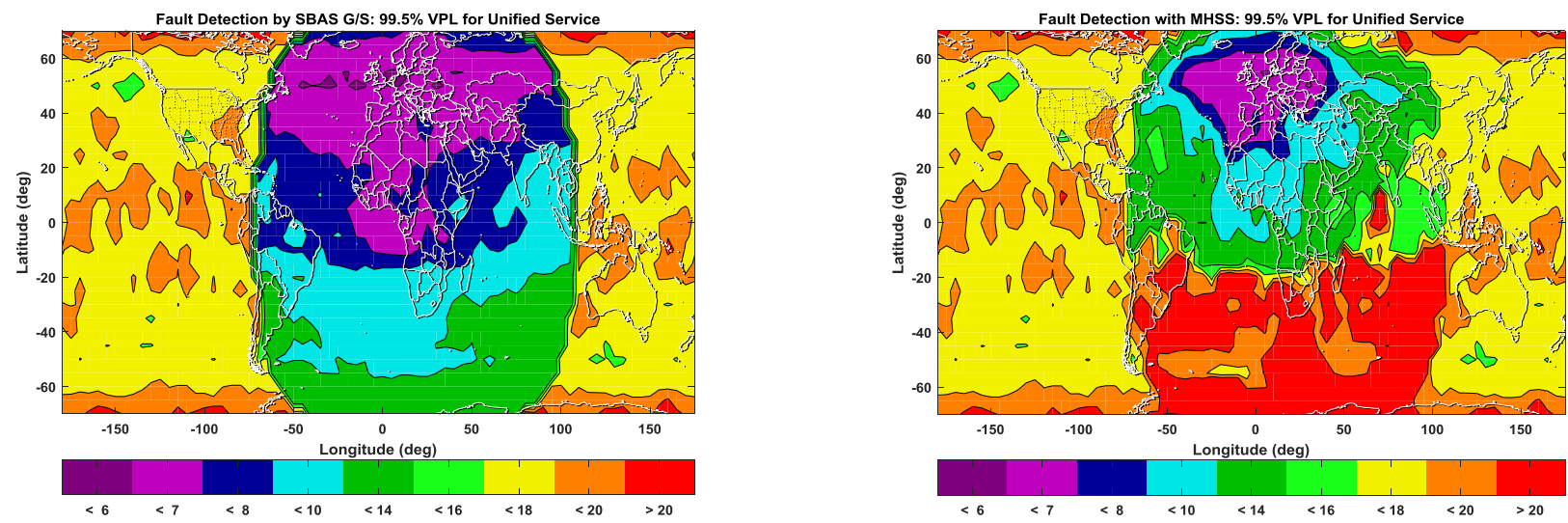

Figure 5: VPL Performance combining SBAS without fault detection and ARAIM. For demonstration purposes both scenarios assume a GEO present to convey the SBAS information to the users, and the same RIMS network. In the plot on the left, SBAS G/S provides fault monitoring to the user. In the plot on the right, fault detection is performed by the user and a prior probability of $10^{-5}$ per approach per satellite is assumed both for ISM and for SBAS.

In this scenario it can be observed that the performance in the southern half of the GEO footprint becomes worse than ARAIM with ISM when the differential corrections and their overbonding are used, but fault detection remains within the user algorithm. The cause is the simplified UDRE model in this simulation. In a real SBAS scenario the effect would be similar, since the UDRE outside the reference station can be higher than a globally provided URA from ISM. In such a combined mode, MHSS could choose between ISM and SBAS overbounding based on best performance.

\section{DISCUSSION}

In the previous sections the method of a combined, unified integrity service based on MHSS has been introduced and several use cases have been confirmed by means of simulation. The present work is only an initial take on an integrity service outside the existing paradigms of SBAS or ARAIM. Two relevant aspects of implementation, both technical and of governance nature are discussed below.

\section{Liability and institutional assignment of the unified service}

Provision of integrity services is not only a technical challenge - aviation operators use a service based on standards, regulations and legislation applicable both to an operator and/or a particular airspace. Having an integrity service depend on multiple secondary systems dilutes liability and may be unacceptable for the airspace authority.

A mechanism allowing an operator of one of the sub-services (ISM or SBAS) to govern usage of such a combined approach can be established if the user is mandated to check for a "use flag" in the corresponding data. As an example, WAAS could provide a flag related to the use of Galileo with an ISM coming from a third party provider. WAAS ground segment could then monitor the performance and disable ISM (and thus, combined ARAIM/SBAS use) in their service volume if necessary.

\section{Clock states}

ARAIM users solve the position equation including a user clock offset to one or multiple GNSS constellations. SBAS users solve the position equation with a clock offset relating to the SBAS system time. If users want to include both SBAS and nonSBAS ranges in their solution it must be assumed that the SBAS corrected measurements reference a different clock than the stand-alone measurements. Additional clock states can be included in the design matrix but will reduce the degree of overdetermination of the linearized system of equations. This entails that the performance in mixed geometries will be slightly degraded if additional clock states are computed.

\section{CONCLUSION}

This paper introduced a novel integrity mechanism that aims to unify the user-level algorithm for SBAS based integrity and ARAIM based integrity. It demonstrated along three use cases that the user integrity performance can be improved and operational benefits regarding continuity can be achieved. Finally, the proposed combination allows architectural changes in the future such as a partial transition of integrity functions from SBAS to the user level, such as the fault detection function which currently depends on the use of GEOs. 


\section{DISCLAIMER}

The work carried out in this paper was performed while the second author was employed at the Institute of Communication and Navigation at the German Aerospace Center (DLR). The technical information contained in this document does not represent any official Airbus Defence \& Space GmbH position.

\section{REFERENCES}

[1] “EGNOS SoL service roadmap,” 2018. [Online]. Available: https://egnos-user-support.essp-sas.eu/new_egnos_ops/system/files/sol_Roadmap.pdf

[2] U.S.-EU Agreement on GPS-Galileo Cooperation, "GPS-Galileo Working Group C ARAIM Technical Subgroup Milestone 3 Report," 2016.

[3] S. Perea, M. Meurer, M. Rippl, B. Belabbas, and M. Joerger, "URA/SISA analysis for GPS and Galileo to support ARAIM," NAVIGATION, vol. 64, no. 2, pp. 237-254, 2017.

[4] S. Perea, M. Meurer, I. Martini, M. Rippl, and B. Pervan, "ARAIM ground architecture based on GNSS monitoring infrastructures," in Proceedings of the 30th International Technical Meeting of The Satellite Division of the Institute of Navigation, no. 2, 2017, pp. 1008-1018.

[5] WAAS T\&E Team, "Global Positioning System (GPS) Standard Positioning Service (SPS) performance analysis report \#102," FAA William J. Hughes Technical Center, Tech. Rep., 2018. [Online]. Available: http://www.nstb.tc.faa.gov/reports/2018_Q2_SPS_PAN102_v1.0.pdf

[6] NSTB/WAAS T\&E Team, "Wide area augmentation system performance analysis report," FAA William J. Hughes Technical Center, techreport, 2018. [Online]. Available: http://www.nstb.tc.faa.gov/reports/waaspan65.pdf

[7] ESSP, "Monthly performance report august 2018," Tech. Rep., 2018. [Online]. Available: https://egnos-usersupport.essp-sas.eu/new_egnos_ops/sites/default/files/documents/88\%20-\%20Monthly\%20Performance\%20Report\%20$\% 20$ August $\% 202018 . p d f$

[8] S. Wallner and F. Amarillo, "ARAIM architecture, presentation at WG-C ARAIM Technical Subgroup Meeting," Tech. Rep., 2013.

[9] B. S. Pervan, S. P. Pullen, and J. R. Christie, “A Multiple Hypothesis Approach to Satellite Navigation Integrity," Journal of The Institute of Navigation, vol. 45, no. 1, 1998.

[10] T. Walter and P. Enge, "Weighted RAIM for Precision Approach," in Proceedings of the ION GNSS Conference 1995. ION, 1995. [Online]. Available: http://waas.stanford.edu/papers/wraim_tfw95.pdf

[11] J. Blanch, T. Walker, P. Enge, Y. Lee, B. Pervan, M. Rippl, A. Spletter, and V. Kropp, "Baseline advanced RAIM user algorithm and possible improvements," IEEE Transactions on Aerospace and Electronic Systems, vol. 51, no. 1, pp. 713732, January 2015.

[12] RTCA/DO-229D: WAAS Minimum Operational Performance Specification (MOPS), RTCA Special Committee 159 Std., 1991.

[13] EUROCAE WG-62, "Draft ED-259 " Minimum Operational Performance Standards for Galileo - Global Positioning System - Satellite-Based Augmentation System Airborne Equipment"," EUROCAE, Tech. Rep., 2018. [Online]. Available: https://eurocae.sharepoint.com/sites/Portal/SitePages/open\%20consultations.aspx

[14] S. Jan, W. Chan, T. Walter, and P. Enge, "Matlab Simulation Toolset for SBAS Availability Analysis," in Proceedings of the ION GNSS Conference 2001, 2001. 